\title{
Quantum Black Holes as Solvents
}

\author{
Erik Aurell $^{1,2} \cdot$ Michał Eckstein $^{3,4}$ D $\cdot$ Paweł Horodecki ${ }^{5,6}$
}

Received: 3 July 2020 / Accepted: 3 April 2021 / Published online: 24 April 2021

(C) The Author(s) 2021

\begin{abstract}
Almost all of the entropy in the universe is in the form of Bekenstein-Hawking (BH) entropy of super-massive black holes. This entropy, if it satisfies Boltzmann's equation $S=\log \mathcal{N}$, hence represents almost all the accessible phase space of the Universe, somehow associated to objects which themselves fill out a very small fraction of ordinary three-dimensional space. Although time scales are very long, it is believed that black holes will eventually evaporate by emitting Hawking radiation, which is thermal when counted mode by mode. A pure quantum state collapsing to a black hole will hence eventually re-emerge as a state with strictly positive entropy, which constitutes the famous black hole information paradox. Expanding on a remark by Hawking we posit that BH entropy is a thermodynamic entropy, which must be distinguished from information-theoretic entropy. The paradox can then be explained by information return in Hawking radiation. The novel perspective advanced here is that if $\mathrm{BH}$ entropy counts the number of accessible physical states in a quantum black hole, then the paradox can be seen as an instance of the fundamental problem of statistical mechanics. We suggest a specific analogy to the increase of the entropy in a solvation process. We further show that the huge phase volume $(\mathcal{N})$, which must be made available to the universe in a gravitational collapse, cannot originate from the entanglement between ordinary matter and/or radiation inside and outside the black hole. We argue that, instead, the quantum degrees of freedom of the gravitational field must get activated near the singularity, resulting in a final state of the 'entangled entanglement' form involving both matter and gravity.
\end{abstract}

Keywords Black holes · Quantum information · Hawking radiation

\footnotetext{
This work was supported by the International Center for the Theory of Quantum Technologies (ICTQT, Gdańsk, Poland) and by the Foundation for Polish Science through TEAM-NET project (Contract No. POIR.04.04.00-00-17C1/18-00) (EA). The work of ME was supported by the National Science Centre in Poland under the research Grant Sonatina (2017/24/C/ST2/00322). The work of $\mathrm{PH}$ was supported by the Foundation for Polish Science through IRAP project co-financed by the EU within the Smart Growth Operational Programme (Contract No. 2018/MAB/5).
}

Michał Eckstein michal@eckstein.pl

Extended author information available on the last page of the article 


\section{Black Hole Information Paradox}

The black hole information paradox can be formulated as the following Gedan- kenexperiment. Assume that quantum mechanics is universally valid, that gravitation is switched off, and that a sufficiently large amount of matter can be found in a pure quantum state. If gravitation is switched on this matter will collapse into a black hole, which by unitarity will still be in a pure quantum state. At a later time this state will evolve to the remainder of the black hole and the Hawking radiation escaping to infinity. Taken separately, neither the remainder black hole nor the escaping radiation are pure; the black hole state is supposed to tend to a maximally mixed state on all black hole states with the same mass, and the radiation is assumed to be thermal. Then, when the black hole evaporation reaches its end, there is no black hole left, but only the radiation, which by assumption has strictly positive entropy. Hence a pure state has evolved into a mixed state, which would break the unitary evolution in quantum mechanics $[1,2]$. The topic has been reviewed multiple times, recently in [3-5] and [6]. The basic proposals for resolving the paradox were summarized in [7] or, more recently, [8] as fundamental information loss, remnants, and information return in the Hawking radiation. In this context "firewalls" and other scenarios involving new physics at the horizon fall into the class of remnants since information is not destroyed and also does not leave the vicinity of the black hole.

In this paper we propose a resolution in the direction of information return, but in the framework of open quantum systems. Our focus here is not on how information actually returns in the Hawking radiation, a topic to which we hope to return in the future, but what are its implications on the concept of entropy. A previous argument in the same vein was voiced by Stephen Hawking in [9] but, to our best knowledge, it had no resonance in the literature. Part of our motivation in this paper is hence to bring attention to this note by Hawking, and to place it in the context of discussions of fundamental concepts of statistical mechanics.

The literature on quantum black holes is very large, and it is not possible to refer to all relevant contributions in an regular article. To guide the reader we here provide a partial summary, for more extensive discussions we refer to the recent reviews e.g. [3-5]. First, as we pursue an approach of information return we will throughout assume that quantum mechanics is universally valid. This means that we have to assume that gravitation is also quantised. In our proposal of quantum entanglement in black hole formation we assume, however, that without black holes the gravitational field is not entangled with other quantum systems. From this standpoint the black hole information paradox is explained, at the kinematic level, by entanglement between the gravitational field and other degrees of freedom during the collapse process. As there is no established and generally accepted theory of quantum gravity we adopt a theory-independent approach (cf., for instance, $[10,11])$. In other words, rather than providing an explicit physical mechanism, which would provide much more detailed understanding, we try to separate out the features which any theoretical model of this kind must have. We hence leave the interesting questions on the gravitational side, in particular those related to the localization of the Hawking radiation [12-14], for a future study. 
The possibility of information return was first proposed by Page [15], and later developed by him in several publications, e.g. [16]. The core problem is that if information is preserved, then the final state of the Hawking radiation, when the entire black hole has radiated away, has to be pure. Since the states of all separate modes of Hawking radiation are thermal this raises a compatibility issue between the total purity and the reduced mixed states (cf. [17]). Furthermore, the difference must be encoded in quantum correlations between the separate Hawking particles. But this is problematic, because these are, for the most part, emitted one by one [18] and do not interact directly. The related issues are discussed to this day: some notable contributions were made by Preskill [19] (and later), Braunstein, Pirandola and Życzkowski [20]; Bradler and Adams [21]; Lochan and Padmanaban [22]; Grudka and collaborators [23]; and very recently by Marolf and Maxfield [24]. More contributions in these directions will be cited further on.

In this paper we will discuss the black hole information problem, under the assumption of a global unitary evolution, as an instance of what is often called the "fundamental problem of statistical mechanics". The latter is the question, dating back to Boltzmann, on how conservative classical dynamics can be reconciled with the increase of entropy and tendency to thermalization, in other words, with the Second Law. The quantum black hole problem has unusual features pertaining to the fact that the phenomena involved are quantum and that the process itself involves unknown constituents. The latter is so since a quantum black hole presumes quantum gravity in the strong-field regime near the singularity, and there is no generally accepted theory for such phenomena. However, we find nonetheless that general arguments of statistical mechanics do shed light on these issues, as first noted by Hawking [9].

In ordinary room-temperature physics a low-entropy crystalline solid (solute) dissolves into a high-entropy liquid (solvent), in the process called solvation. We propose that the massive increase in the entropy during the gravitational collapse is a physical process analogous to solvation, where the singularity dissolves the degrees of freedom of the gravitational field.

The paper is organized as follows. In Sect. 2 we survey well-known results on the Bekenstein-Hawking entropy, emphasizing the fact that it is by far the largest part of entropy in the Universe. In Sect. 3 we present our proposal of considering the Bekenstein-Hawking entropy as the entanglement entropy between the black hole and the quantum gravitational field, and work out some simple consequences of such a hypothesis. ${ }^{1}$ In Sect. 4 we present our second argument about the relation of the black hole information paradox to the foundational problem of statistical mechanics, and in Sect. 5 we sum up and discuss the results.

\footnotetext{
1 After completion of this work we found that mechanisms involving entanglement between matter and gravitational field have recently been proposed by Requardt [63] and Kay [64], but from different starting points, and with little overlap with our approach.
} 


\section{The Entropy Increase}

The Bekenstein-Hawking (BH) entropy of a black hole is $S_{\mathrm{BH}}=k_{B} \frac{1}{4} \frac{A}{l_{\mathrm{P}}^{2}}$, where $A$ is the surface area of the horizon and $l_{\mathrm{P}}=\sqrt{\frac{\hbar G}{c^{3}}}$ is the Planck length $\left(1.6 \cdot 10^{-35} \mathrm{~m}\right)$. The Boltzmann's constant $k_{B}=1.38 \cdot 10^{-23} \frac{J}{K}$ can be set to one by choosing a suitable temperature scale. In the following we will, however, keep it explicit.

Most astrophysical black holes are approximately stationary, have low rotation, as compared to the extreme allowed by the Kerr geometry, and are electrically neutral. The space-time around such a black hole is thus well approximated by the spherically symmetric Schwarzschild geometry, where the radius of the trapping horizon is determined by its mass, $R=\frac{2 G M}{c^{2}}$. The latter can also be written as $R=2 l_{\mathrm{P}} \frac{M}{m_{\mathrm{P}}}$ where $m_{\mathrm{P}}=\sqrt{\frac{c \hbar}{G}}$ is the Planck mass $\left(2 \cdot 10^{-8} \mathrm{~kg}\right) . \mathrm{BH}$ entropy of a Schwarzschild black hole is therefore also $S_{\mathrm{BH}}=k_{B} 4 \pi \frac{M^{2}}{m_{\mathrm{P}}^{2}}$. In ordinary thermodynamics entropy is an extensive quantity, i.e. proportional to mass. The black hole entropy is, on the other hand, super-extensive-it increases faster than linearly with the mass. One simple consequence of this fact is the following: denote by $E_{\mathrm{BH}}=M c^{2}$ the energy of a black hole. According to a standard thermodynamical formula the inverse temperature is the partial derivative of entropy with respect to energy, $\frac{1}{T}=\frac{\partial S}{\partial E}$. After inserting $S_{\mathrm{BH}}$ and $E_{\mathrm{BH}}$ from above we obtain $\frac{\partial S_{\mathrm{BH}}}{\partial E_{\mathrm{BH}}}=\frac{4 \pi k_{B}}{m_{\mathrm{P}}^{2} c^{2}} \frac{\partial M^{2}}{\partial M}$. This gives a temperature, which is not a free parameter, but which decreases with mass as $T_{\mathrm{BH}}=\frac{m_{\mathrm{P}} c^{2}}{8 \pi k_{B}}\left(\frac{M}{m_{\mathrm{P}}}\right)^{-1}$. This temperature, which is the temperature of the Hawking radiation, expressed in the units of Planck energy $\left(m_{\mathrm{P}} c^{2} \approx 2 \cdot 10^{9} \mathrm{~J}\right)$, is weighted down by the mass of the black hole in Planck mass units.

It is also well established that $\mathrm{BH}$ entropy increases faster with mass than any matter or light which could have formed the black hole [25]. For example, for a photon gas we would have $S \sim M^{\frac{3}{2}}[4,26]$, while $S_{B H} \sim M^{2}$. Hence, it is clear that the $\mathrm{BH}$ entropy is a very special type of entropy, probably unique in our Universe. Furthermore, it not only increases faster with mass than anything else, it is also in total much larger than anything else. Since the BH entropy is super-extensive most of this type of entropy should be associated with the largest objects in the Universe. The largest such objects known are the super-massive black holes found in the center of galaxies; the fraction of the Universe's entropy not inside a super massive black hole has been estimated to be as small as one part in $10^{7}$, cf. Table 1 in [27].

In Boltzmann's theory of thermodynamics, entropy is a measure of the available phase space $S=k_{B} \log \mathcal{N}$. It is startling and perhaps paradoxical that supermassive black holes, which occupy a very small volume of our Universe seem to contain almost all the available phase space. If this is so, it is crucial to understand what is this phase space and how it becomes accessible to the Universe via the creation of a black hole. 


\section{Entanglement in Black Hole Formation}

Consider an astrophysical black hole formed from some part of the universe which was in a mixed state as far back as we can know. Before the collapse it can hence be represented as a part of a larger pure state

$$
\left|\Psi^{(0)}\right\rangle_{U}=\sum_{i} a_{i}|i\rangle_{S}|i\rangle_{A}
$$

where $U$ stands for "universe", $S$ stands for "system" (or "star") and $A$ stands for the rest of the universe that here plays the role of an "ancilla". The von Neumann entropy of $S$ is the same as the entropy of $A$,

$$
S\left[\operatorname{Tr}_{A}\left(\left|\Psi^{(0)}\right\rangle_{U}\left\langle\left.\Psi^{(0)}\right|_{U}\right)\right]=-\sum_{i}\left|a_{i}\right|^{2} \log \left|a_{i}\right|^{2}=S\left[\operatorname{Tr}_{S}\left(\left|\Psi^{(0)}\right\rangle_{U}\left\langle\left.\Psi^{(0)}\right|_{U}\right)\right],\right.\right.
$$

and it measures the entanglement between $S$ and $A$. After the formation of the black hole one would usually expect to obtain the state

$$
\left|\Psi^{(1)}\right\rangle_{U}=\sum_{i} b_{i}|i\rangle_{B H}|i\rangle_{A^{\prime}}
$$

where $A^{\prime}$ stands for the rest of the universe outside the black hole, and where the entropy of the black hole $(B H)$ is much larger than the entropy of $S$. The gravitational collapse is essentially a local phenomenon, which starts when a (sufficiently massive) star losses its thermal equilibrium. Hence, the "universe" here means some neighborghood of the initial star, the size of which is limited by the finite speed of propagation of signals and physical interactions.

It is a fundamental result of quantum information theory that entanglement cannot increase under local operations and classical communication (LOCC) [28]. This means that two experimentalists who operate on spacelike-separated parts of a system, and who do not share a quantum resource and can only communicate classically to one another, cannot increase the entanglement between the two parts, whatever they do. They can only decrease it. In fact, they can trivially eliminate the entanglement almost completely by letting one or both parts decohere in contact with a bath. In order to increase entanglement the two experimentalists and the system must act as one large quantum system. Similarly in natural systems, entanglement between two parts can only increase if these parts interact, either directly, or through an intermediary.

For the entropy of the system to increase massively, the quantum matter forming the black hole would therefore have to massively interact with the rest of the universe during the collapse process, and it cannot do so with the surrounding ordinary matter and light through accretion disks, radiation, etc. since all these would bring in only a tiny fraction of the required entropy. In other words, the von Neumann entropy of a black hole, i.e. 


$$
S\left(\rho_{B H}\right)=-\sum_{i}\left|b_{i}\right|^{2} \log \left|b_{i}\right|^{2}, \text { with } \rho_{B H}=\operatorname{Tr}_{A^{\prime}}\left(\left|\Psi^{(1)}\right\rangle_{U}\left\langle\left.\Psi^{(1)}\right|_{U}\right)\right.
$$

should not be very much larger than the von Neumann entropy of the initial system (2). Since in fact BH entropy is much greater than the entropy of the collection of the initial constituents, we must assume that the local unitary transformations involve not only the constituents themselves but also some additional local parts carrying extra quantum degrees of freedom. Though theoretical models here greatly outweigh the facts, it appears reasonable to assume that these unknown parts are related to the quantumness of the gravitational field, cf. [29]. Such an assumption could be studied from various perspectives, for instance string theory [6, 30], loop quantum gravity [31-33] or analogue gravity [34]. Here we adopt a model-independent approach, basing solely on the general principles of quantum information (cf. [35]).

Consequently, we should extend the initial quantum description (1) to encompass the quantum degrees of freedom of the gravitational field. We assume that initially the gravitational field is not appreciably entangled with the other degrees of freedom. By linearity of quantum mechanics it then suffices to consider the case when the gravitational field is pure. Hence, instead of (1) the full initial quantum state before the collapse is

$$
\left|\tilde{\Psi}^{(0)}\right\rangle_{U+G F}=\left|\Psi^{(0)}\right\rangle_{U}\left|\Phi^{(0)}\right\rangle_{G F}
$$

where $G F$ stands for the gravitational field. Now, the local unitary evolution introduced above does not, by assumption, change the entropy of the outside part of the universe $A^{\prime}$. On the other hand, it can contribute to the massive entropy increase in a black hole by an interaction that leads to the final state:

$$
\left|\widetilde{\Psi}^{(1)}\right\rangle_{U+G F}=\sum_{i} b_{i}\left[\sum_{k} c_{k}^{i}|k\rangle_{B H}|k\rangle_{G F}\right]|i\rangle_{A^{\prime}}
$$

States of this type are known in quantum information theory as "entangled entanglement" $[36,37]$. Contrary to ordinary entanglement which involves one sum over a Hilbert-Schmidt decomposition of a bipartite state, there is now a double sum. In consequence the entanglement between any two subsystems is a property, which itself is entangled with the third subsystem. In the case at hand the internal $\mathrm{BH}+G \mathrm{~F}$ entanglement, conditioned at least upon some of the states of the rest of the Universe $A^{\prime}$, can then be much greater than the one between the joint system $B H+G F$ and the rest of the Universe $A^{\prime}$ - for an illustration, see Fig. 1. Indeed, with

$$
\tilde{\rho}_{B H}=\operatorname{Tr}_{A^{\prime}+G F}\left(\left|\widetilde{\Psi}^{(1)}\right\rangle_{U+G F}\left\langle\left.\widetilde{\Psi}^{(1)}\right|_{U+G F}\right)=-\sum_{i}\left|b_{i}\right|^{2} \sum_{k}\left|c_{k}^{i}\right|^{2}|k\rangle_{B H}\left\langle\left. k\right|_{B H}\right.\right.
$$

we have

$$
S\left(\widetilde{\rho}_{B H}\right)=-\sum_{k, i}\left|b_{i}\right|^{2}\left|c_{k}^{i}\right|^{2} \log \left(\sum_{j}\left|b_{j}\right|^{2}\left|c_{k}^{j}\right|^{2}\right)
$$




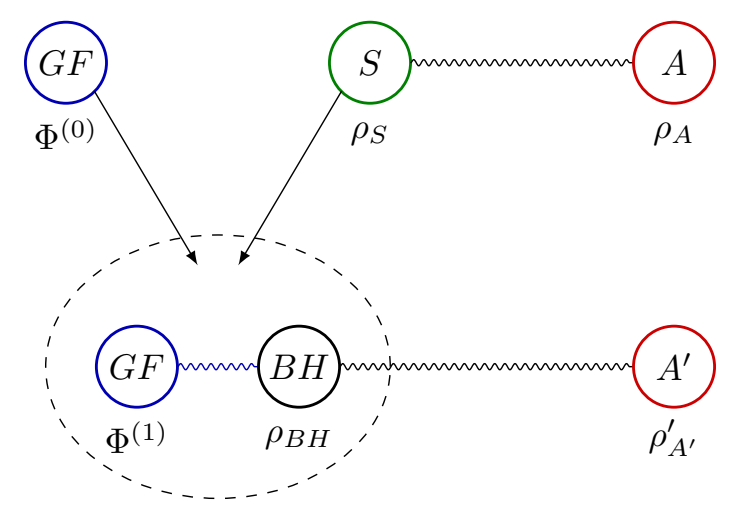

Fig. 1 The suggested picture of the process of black hole formation involving entanglement with the quantum degrees of freedom of the gravitational field, initially assumed to be in a pure state $\left|\Phi^{(0)}\right\rangle_{G F}$ (see text). Both the system $S$, i.e. the constituents that are to collapse, as well as the rest of the universe $A$, have small initial von Neumann entropies $S\left(\rho_{S}\right)=S\left(\rho_{A}\right)=-\sum_{i}\left|a_{i}\right|^{2} \log \left|a_{i}\right|^{2}$. Since the gravitational collapse is a local process it can be seen as a local unitary operation $U$ which leads to the interaction of the system of constituents, the gravitational field and some limited part of the rest of the universe. The final state representing entangled entanglement results in the black hole's entropy (4) much higher than the initial entropy of the constituents (2). This is precisely the effect of interaction with the gravitational field which, initially, was not entangled with the other degrees of freedom

The value of $S\left(\widetilde{\rho}_{B H}\right)$ can easily become much larger than that of the initial configuration given by (2). Roughly, one can estimate (by assuming uniform distributions, $\left|a_{i}\right|^{2}=1 / d_{S}$ and $\left.\left|c_{k}^{i}\right|^{2}=1 / d_{G F}\right)$ that $S\left(\rho_{S}\right) \sim \log d_{S}$ and $S\left(\widetilde{\rho}_{B H}\right) \sim \log d_{G F}$, with $d_{S}$ and $d_{G F}$ denoting the numbers of active modes of the star and the gravitational field, respectively. Hence, the price to pay for avoiding the black hole information paradox is to assume that the gravitational collapse activates a huge number, $d_{G F} \gg d_{S}$, of quantum gravitational degrees of freedom, which remain idle if there is no black hole.

Finally, let us note that in the process of the black hole evaporation, the quantum correlations in the $B H+G F$ system can be transferred to the entanglement of the combined $B H+G F+H R$ system, where $H R$ stands for the Hawking radiation. Eventually, when the black hole has radiated away completely, as it would do in a scenario with information return, there is only the Hawking radiation left, the modes of which are entangled. The question of how this can actually happen is, however, outside of the scope of the present work. For some further kinematic considerations, see [17].

\section{Black Hole Information Paradox as an Instance of the Foundational Problem of Statistical Mechanics}

If the BH entropy satisfies Boltzmann's formula $(S=\log \mathcal{N})$, then the formation of a black hole makes accessible to the universe a number $\mathcal{N} \sim e^{4 \pi \frac{M^{2}}{m_{\mathrm{P}}^{2}}}$ of states which were previously inaccessible. In the language of quantum information $S_{\mathrm{BH}}$ would be the log of the dimension of the subspace of Hilbert space needed to describe 
the interior of a black hole. General arguments have been advanced against this socalled strong interpretation of BH entropy, cf. [38].

However, such arguments are somewhat troublesome in the light of modern statistical mechanics which strongly favours the objective interpretation where entropy is associated with real degrees of freedom, and it is not only a measure of our ignorance. Many papers on black hole thermodynamics and information paradox are written in the subjective tradition of thermodynamics founded on the max-entropy principle by Edwin T. Jaynes [39, 40]. The latter is invoked to draw conclusions about a complex system from a limited number of observables. Yet, Jaynes' principle was shown to lead to incorrect predictions when the studied phenomenon is not in equilibrium [41], which is certainly the case of the gravitational collapse. Furthermore, Jaynes' principle leads to wrong conclusions about the entanglement [42], and must hence be considered incompatible with modern quantum information theory, where entanglement is fundamental [28].

In order to make a connection with thermodynamics, we first need to distinguish between entropy in the thermodynamic and the information-theoretic sense. In the first case, following [9], BH entropy is assumed to be the log of the number of quantum states of the black hole with the same mass. In the second case, as discussed above around formulas (1) and (3), BH entropy is the von Neumann entropy measuring entanglement. The perceived problem is that the first can be much larger than the second. This can, however, be seen as a reformulation of the foundational problem of statistical mechanics as posed by Thompson, Maxwell and Boltzmann [43]. In other words, the problem is that the conservative classical dynamics preserves entropy while many systems appear to behave as if tending to thermodynamic equilibrium and then the entropy increases - the Second Law applies. A widely studied analogous problem is how high-dimensional quantum systems can behave as if they thermalize, the "eigenstate thermalization hypothesis" [44-47]. For a further recent study in an explicitly gravitational context, see [48]. An ensemble of classical deterministic systems will, if the dynamics can be followed exactly, preserve Shannon entropy (von Neumann entropy in the quantum case), but will nevertheless after relaxation behave as if in thermal equilibrium, in an ensemble with the thermodynamic entropy. As argued by Khinchin [49, 50] the systems under consideration in statistical mechanics have very high dimensionality, and the kinds of observations that can be made on them are simple. Both these properties hold for black holes: the dimensionality is extremely high, and the observations (or undisputed theoretical predictions) that can be made are quite limited.

There is thus no paradox in the fact that the thermodynamic entropy of the Hawking radiation, estimated by summing the contributions of all the modes, is much larger than the von Neumann entropy of the Hawking radiation. For a black hole that starts out in a pure state and eventually evaporates, the time-evolution of its von Neumann entropy follows the Page curve $[16,51]$ and finally drops to zero.

The total process whereby a collapsing star (in a pure quantum state) eventually turns into photons and other Hawking particles streaming away to infinity can be unitary (given an appropriate theory of quantum gravity) and would then not increase the von Neumann entropy, but would be indistinguishable (by the measurements that can practically be made) from a process that actually does increase 
entropy, and which actually is not unitary. Indeed, an observer independent from the system can effectively be coupled only to a few (collective) degrees of freedom. In order to observe that the actual von Neumann entropy of the system in question is small one would have to couple to (almost) all degrees of freedom and then unentangle them (i.e. effectively diagonalise the system's density matrix)—either via tomography and subsequent extensive (classical) post-processing, or physicallyby accumulating the small von Neumann entropy on a small part of the degrees of freedom, leaving the others pure. The latter process is a generalised version, involving memory effects, of the standard concept of algorithmic cooling [52]. Hence, the observer would become an analogue of Maxwell's daemon who resets the temperature to much a lower value, corresponding to the von Neumann entropy [53].

The novelty of our argument, which was previously stated en passant by Hawking in [9], is to point out that this process also occurs in ordinary matter and has been at the forefront of research in statistical mechanics since the 19th century. While quantum black holes hold many other mysteries, this is not one of them.

\section{Quantum vs Classical Gravity}

It is commonplace to assume that the resolution of the black hole information paradox requires a major departure from unitary quantum mechanics [8] and/or the breakdown of the structure of relativistic spacetime [23, 54]. Our conclusion is that the information contained in the infalling matter is not lost, but remains concealed in the quantum degrees of freedom of the gravitational field which was initially not entangled with the matter. At the same time those quantum degrees of freedom must be very thoroughly mixed with those of the matter which fell into the black hole earlier. Consequently, it is indeed possible for a quantum black hole to have a very large thermodynamic entropy, much larger than the entanglement entropy (von Neumann entropy) of all the initial matter.

The picture is analogous to the process of solvation: the black hole dissolves the solute-the infalling matter along with the corresponding gravitational field, which have relatively low entropy. The resulting solution will, on the other hand, have a relatively huge entropy. The very large increase in the entropy suggests that solubility is very large - the solute is practically infinitely soluble in the solvent.

We should confront the present analysis with the fact that the gravitational field, say that of the Earth, acts on the particles unitarily via an external potential. An analogous issue occurs in quantum optics: we know that whereas strong laser fields are fundamentally of quantum nature, they act on test quantum particles as effectively classical fields, via a time-dependent Hamiltonian. The fundamental difference is that the gravitational field, which affects the quantum particles in a classical way is not strong at all. One could claim that big masses emit a large number of gravitons, corresponding to the quantum degrees of freedom of the field, which decohere on the way to the object. If there is a sufficiently large number of them (e.g. macroscopic objects) the internal nature of the quantum gravitational field would lead to the emission of non-virtual long-living quanta, which could travel for long distances. 
A related issue is the expectation $[55,56]$ that small particles may entangle with each other via the gravitational field (cf. [57-60]). In this case, the gravitons are likely to be virtual (cf. [61]). They do not exist independently and, as such, they do not contribute to the entropy, while the interaction they cause is unitary. The physics near the black hole singularity is quite different. The huge mass concentration activates the genuine, "non-virtual", quantum degrees of freedom of the gravitational field.

In the presented analysis we have adopted a purely quantum information-theoretic approach, which is model-independent. Its advantage is to provide a general kinematic setup for all models of quantum gravity. One specific model of activation of the genuine quantum gravitational degrees of freedom was considered within loop quantum gravity [31-33]. A quite different mechanism involving wormholes was recently put forward in the context of string theory and the AdS/CFT correspondence $[6,24,62]$.

In summary, we believe that the presented analysis offers a compelling explanation of the black hole information paradox and opens a new avenue towards quantum gravity.

Acknowledgements We thank Lárus Thorlacius, Angelo Vulpiani and Rui Li for discussions and useful remarks. We thank Arun Kumar Pati and Sougato Bose for comments on the preprint of this work and for drawing our attention to refs. [35] and [61], respectively. We also thank the anonymous Referees for their pertinent comments.

Open Access This article is licensed under a Creative Commons Attribution 4.0 International License, which permits use, sharing, adaptation, distribution and reproduction in any medium or format, as long as you give appropriate credit to the original author(s) and the source, provide a link to the Creative Commons licence, and indicate if changes were made. The images or other third party material in this article are included in the article's Creative Commons licence, unless indicated otherwise in a credit line to the material. If material is not included in the article's Creative Commons licence and your intended use is not permitted by statutory regulation or exceeds the permitted use, you will need to obtain permission directly from the copyright holder. To view a copy of this licence, visit http://creativecommons.org/licen ses/by/4.0/.

\section{References}

1. Hawking, S.: Breakdown of predictability in gravitational collapse. Phys. Rev. D 14, 2460 (1976)

2. Hawking, S., Perry, M., Strominger, A.: Soft hair on black holes. Phys. Rev. Lett. 116, 231301 (2016)

3. Verlinde, E., Verlinde, H.: Black hole entanglement and quantum error correction. J. High Energy Phys. 10, 107 (2013)

4. Harlow, D.: Jerusalem lectures on black holes and quantum information. Rev. Mod. Phys. 88, 15002 (2016)

5. Marolf, D.: The black hole information problem: past, present and future. Rep. Progr. Phys. 80, 092001 (2017)

6. Almheiri, A., Hartman, T., Maldacena, J., Shaghoulian, E., Tajdini, A.: The entropy of Hawking radiation, arXiv:2006.06872 (2020)

7. Giddings, S.B.: Comments on information loss and remnants. Phys. Rev. D 49, 4078 (1998)

8. Unruh, W.G., Wald, R.M.: Information loss. Rep. Prog. Phys. 80, 092002 (2017)

9. Hawking, S.: Information preservation and weather forecasting for black holes, arXiv:1401.5761 (2014) 
10. Brunner, N., Cavalcanti, D., Pironio, S., Scarani, V., Wehner, S.: Bell nonlocality. Rev. Mod. Phys. 86, 419 (2014)

11. Popescu, S.: Nonlocality beyond quantum mechanics. Nat. Phys. 10, 264 (2014)

12. Hotta, M., Schützhold, R., Unruh, W.G.: Partner particles for moving mirror radiation and black hole evaporation. Phys. Rev. D 91, 124060 (2015)

13. Wald, R.M.: Particle and energy cost of entanglement of Hawking radiation with the final vacuum state. Phys. Rev. D 100, 065019 (2019)

14. Good, M.R.R., Linder, E.V., Wilczek, F.: Moving mirror model for quasithermal radiation fields. Phys. Rev. D 101, 025012 (2020)

15. Page, D.N.: Is black-hole evaporation predictable? Phys. Rev. Lett. 44, 301 (1980)

16. Page, D.N.: Information in black hole radiation. Phys. Rev. Lett. 71, 3743 (1993)

17. Aurell, E., Eckstein, M., Horodecki, P.: Quantum information in Hawking radiation, arXiv:2012. 14418 (2020)

18. Gray, F., Schuster, S., Van-Brunt, A., Visser, M.: The Hawking cascade from a black hole is extremely sparse. Class. Quantum Grav. 33, 115003 (2016)

19. Preskill, J.: Do black holes destroy information?, Int. Symp. on Black Holes, Membranes, Wormholes, and Superstrings, arXiv:hep-th/9209058 (1992)

20. Braunstein, S.L., Pirandola, S., Życzkowski, K.: Better late than never: information retrieval from black holes. Phys. Rev. Lett. 110, 101301 (2013)

21. Bradler, K., Adami, C.: The capacity of black holes to transmit quantum information. J. High Energy Phys. 05, 095 (2014)

22. Lochan, K., Padmanabhan, T.: Extracting information about the initial state from the black hole radiation. Phys. Rev. Lett. 116, 051301 (2016)

23. Grudka, A., Hall, M.J.W., Horodecki, M., Horodecki, R., Oppenheim, J., Smolin, J.A.: Do black holes create polyamory? J. High Energy Phys. 11, 045 (2018)

24. Marolf, D., Maxfield, H.: Observations of Hawking radiation: the Page curve and baby universes, arXiv:2010.06602 (2020)

25. Bekenstein, J.D.: Universal upper bound on the entropy-to-energy ratio for bounded systems. Phys. Rev. D 23, 287 (1981)

26. 't Hooft, G.: Dimensional reduction in quantum gravity, arXiv:gr-qc/9310026 (1993)

27. Egan, C.A., Lineweaver, C.H.: A larger estimate of the entropy of the universe. Astrophys. J. 710, 1825 (2010)

28. Horodecki, R., Horodecki, P., Horodecki, M., Horodecki, K.: Quantum entanglement. Rev. Mod. Phys. 81, 865 (2009)

29. Sorkin, R.D.: The Statistical mechanics of Black Hole Thermodynamics, arXiv:gr-qc/9705006 (1997)

30. Susskind, L., Uglum, J.: Black hole entropy in canonical quantum gravity and superstring theory. Phys. Rev. D 50, 2700 (1994)

31. Perez, A.: No firewalls in quantum gravity: the role of discreteness of quantum geometry in resolving the information loss paradox. Class. Quantum Grav. 32, 084001 (2015)

32. Perez, A.: Black holes in loop quantum gravity. Rep. Prog. Phys. 80, 126901 (2017)

33. Amadei, L., Liu, H., Perez, A.: Unitarity and information in quantum gravity: a simple example, arXiv:1912.09750 (2019)

34. Liberati, S., Tricella, G., Trombettoni, A.: The information loss problem: an analogue gravity perspective. Entropy 21, 940 (2019)

35. Braunstein, S.L., Pati, A.K.: Quantum information cannot be completely hidden in correlations: implications for black hole information paradox. Phys. Rev. Lett. 98, 080502 (2007)

36. Krenn, G., Zeilinger, A.: Entangled entanglement. Phys. Rev. A 54, 1793 (1997)

37. Walther, P., Resch, K.J., Brukner, Č, Zeilinger, A.: Experimental entangled entanglement. Phys. Rev. Lett. 97, 020501 (2006)

38. Hossenfelder, S., Smolin, L.: Conservative solutions to the black hole information problem. Phys. Rev. D 81, 064009 (2010)

39. Jaynes, E.T.: Information theory and statistical mechanics. Phys. Rev. 106, 620 (1957)

40. Jaynes, E.T.: Information theory and statistical mechanics II. Phys. Rev. 108, 171 (1957)

41. Auletta, G., Rondoni, L., Vulpiani, A.: On the relevance of the maximum entropy principle in nonequilibrium statistical mechanics. Eur. Phys. J. Spec. Top. 226, 2327 (2017)

42. Horodecki, R., Horodecki, M., Horodecki, P.: Entanglement processing and statistical inference: the Jaynes principle can produce fake entanglement. Phys. Rev. A 59, 1799 (1999) 
43. Lebowitz, J.L.: Microscopic origins of irreversible macroscopic behavior. Phys. A 263, 516 (1999)

44. Deutsch, J.M.: Quantum statistical mechanics in a closed system. Phys. Rev. A 43, 2046 (1991)

45. Srednicki, M.: Chaos and quantum thermalization. Phys. Rev. E 50, 888 (1994)

46. Rigol, M., Dunjko, V., Olshanii, M.: Thermalization and its mechanism for generic isolated quantum systems. Nature 452, 854 (2008)

47. Reimann, P., Kastner, M.: Equilibration of isolated macroscopic quantum systems. New J. Phys. 14, 043020 (2012)

48. Oltean, M., Bonetti, L., Spallicci, A.D.A.M., Sopuerta, C.F.: Entropy theorems in classical mechanics, general relativity, and the gravitational two-body problem. Phys. Rev. D 94, 064049 (2016)

49. Khinchin, A.I.: Mathematical foundations of statistical mechanics, Courier Corporation, 1 Jan (1949)

50. Chibbaro, S., Rondoni, L., Vulpiani, A.: Reductionism, Emergence and Levels of Reality. Springer International Publishing, New York (2014)

51. Page, D.: Time dependence of Hawking radiation entropy. JCAP 09, 028 (2013)

52. Cleve, R., DiVincenzo, D.P.: Schumacher's quantum data compression as a quantum computation. Phys. Rev. A 54, 2636 (1996)

53. Vedral, V.: Landauer's erasure, error correction and entanglement. Proc. R. Soc. Lond. A 456, 969 (2000)

54. Verlinde, E.: On the origin of gravity and the laws of Newton. J. High Energy Phys. 04, 029 (2011)

55. Bose, S., Mazumdar, A., Morley, G.W., Ulbricht, H., Toros, M., Paternostro, M., Geraci, A., Barker, P., Kim, M.S., Milburn, G.: A spin entanglement witness for quantum gravity. Phys. Rev. Lett. 119, $240401(2017)$

56. Marletto, C., Vedral, V.: Gravitationally-induced entanglement between two massive particles is sufficient evidence of quantum effects in gravity. Phys. Rev. Lett. 119, 240402 (2017)

57. Pikovski, I., Zych, M., Costa, F., Brukner, Č: Universal decoherence due to gravitational time dilation. Nat. Phys. 11, 668 (2015)

58. Belenchia, A., Wald, R.M., Giacomini, F., Castro-Ruiz, E., Brukner, Č, Aspelmeyer, M.: Quantum superposition of massive objects and the quantization of gravity. Phys. Rev. D 98, 126009 (2018)

59. Belenchia, A., Wald, R.M., Giacomini, F., Castro-Ruiz, E., Brukner, Č, Aspelmeyer, M.: Information content of the gravitational field of a quantum superposition. Int. J. Mod. Phys. D 28, 1943001 (2019)

60. Zych, M., Costa, F., Pikovski, I., Brukner, Č: Bell's theorem for temporal order. Nat. Comm. 10, $3772(2019)$

61. Marshman, R.J., Ryan, J., Mazumdar, A., Bose, S.: Locality \& entanglement in table-top testing of the quantum nature of linearized gravity. Phys. Rev. A 101, 052110 (2020)

62. Almheiri, A., Hartman, T., Maldacena, J., Shaghoulian, E., Tajdini, A.: Replica wormholes and the entropy of Hawking radiation. J. High Energy Phys. 05, 013 (2020)

63. Requardt, M.: The incomplete semiclassical analysis of the black hole information paradox and its completion via entanglement of radiation and quantum gravity degrees of freedom, arXiv:1503. 07312 (2015)

64. Kay, B.S.: The matter-gravity entanglement hypothesis. Found. Phys. 48, 542 (2018)

Publisher's Note Springer Nature remains neutral with regard to jurisdictional claims in published maps and institutional affiliations. 


\section{Authors and Affiliations}

\section{Erik Aurell $^{1,2} \cdot$ Michał Eckstein $^{3,4}$ D $\cdot$ Paweł Horodecki ${ }^{5,6}$}

Erik Aurell

eaurell@kth.se

Paweł Horodecki

pawhorod@pg.edu.pl

1 KTH-Royal Institute of Technology, AlbaNova University Center, 10691 Stockholm, Sweden

2 Faculty of Physics, Astronomy and Applied Computer Science, Jagiellonian University, 30-348 Kraków, Poland

3 Institute of Theoretical Physics, Jagiellonian University, ul. Łojasiewicza 11, 30-348 Kraków, Poland

4 Institute of Theoretical Physics and Astrophysics, National Quantum Information Centre, Faculty of Mathematics, Physics and Informatics, University of Gdańsk, Wita Stwosza 57, 80-308 Gdańsk, Poland

5 International Centre for Theory of Quantum Technologies, University of Gdańsk, Wita Stwosza 63, 80-308 Gdańsk, Poland

6 Faculty of Applied Physics and Mathematics, National Quantum Information Centre, Gdańsk University of Technology, Gabriela Narutowicza 11/12, 80-233 Gdańsk, Poland 